\title{
LA CONTEXTUALIZACIÓN DEL CURRÍCULO UN MEDIO PARA POTENCIAR EL DESARROLLO
}

\author{
M.Sc. Flor Mary Azofeifa Castro*
}

La autora explica la experiencia de dar un lugar al curriculo en sudimensión microcultural (escuela) y macrocultural (comunidad) en centros de educación de Primero y Segundo Ciclos de Guápiles, Limón y Pérez Zeledón. Para lograrlo, presenta un marco teórico fundamentado en los postulados constructivistas de D. Ausbel. Asimismo, lleva a cabo una reflexión acerca del uso del portafolio como instrumento de evaluación. La propuesta tiene una visión holística que orienta los procesos educativos hacia la multidisciplinariedad y la transdisciplinariedad.
The author describes the experience of taking into account the curriculum for the microcultural dimension (school) and the macrocultural dimension (community) in primary schools in Gúapiles, Limón and Pérez Zeledón. To achieve this, she presents a theoretical framework based on D. Ausbel's constructivist postulates. The use of portfolios as a tool for evaluation is also analyzed. The proposal has a holistic view orienting educational processes toward multidisciplinary and transdisciplinan. approaches.

* Es bachiller en Educación Preescolar, licenciada en Educación Primaria por la Universidad de Costa Rica y máster en Administración Educativa por la Universidad Central Costarricense. Es profesora y extensionista de la División de Educación Rural, donde ha desarrollado experiencias en las áreas de contextualización curricular y educación no formal. 


\section{Antecedentes}

Esta propuesta se construye durante 1996, en el marco del programa UNIR, que busca nuevas formas de propiciar el desarrollo rural. Es así como la División de Educación Rural participan de dicho programa, en un enlace interinstitucional con el Ministerio de Educación Pública, representado por la Dirección Regional de Guápiles con los asesores específicos y supervisores la elaboraron con base en necesidades diagnosticadas en comunidades rurales, a través de talleres participativos.

La Contextualización del Currículo un Medio para Potenciar el Desarrollo es una alternativa para primero y segundo ciclos de la enseñanza general básica; que pretende transformar la práctica pedagógica en las escuelas rurales; convirtiendo los procesos en oportunidades significativas para las y los estudiantes.

El interés de que la educación formal perfile el desarrollo rural a través de la mediación pedagógica requiere de un concepto más humano y menos productivista-economicista; que se resume como:

"El Desarrollo Rural supone el desarrollo agrario, pero tiene que ver sobre todo con los cambios que ocurren en los niveles sociales y calidad de vida de los hombres y mujercs del campo" (Vega Posada 1998, p. 2).

Se parte de que la educación básica le ofrezca a los niños y las niñas durante los seis años la oportunidad de ejercitarse en lo cotidiano, desarrollando sus habilidades y capacidades con muchacreatividad, criticidad y arraigo.

La fundamentación teórico-filosófica se enmarcada en los postulados constructivistas de D. Ausbel y del currículum integrado. Desde esta concepción, la educación es un proceso que ve al individuo como sujeto de transformación cualitativa y el contenido como objeto de conocimiento no acabado y en constante evolución.

Esta interacción permite visualizar la educación como un proceso individual y social; es decir, no sólo atiende el desarrollo y maduración de la persona en sí misma, sino que promueve la integración de todas las áreas y componentes del currículum, que permite fortalecer esa maduración. Esta tendencia le otorga el carácter socializante que debe tener la educación. Al respecto, Gómez manifiesta:

"No se puede encerrar una Política Curricular dentro de la sala de clases, pues sería la negación del sistema y por consecuencia su muerte. Los sujetos, procesos y elementos deben guardar entre sí una estrecha y permanente relación. Centrando el sistema en el niño, se afecta 
de inmediato a toda la sociedad en sus estructuras y funciones: familia, escuela, iglesia, gobierno y econonia" (Mideplan, 1993, p. 245-252).

La función individual y la social deben percibirse como dos elementos que se relacionan en forma estrecha y constante. Por este motivo interesa rescatar la necesidad de ver el fenómeno educativo desde una perspectiva de interrelación escuela (microcultura) y comunidad (macrocultura).

El componente sociocultural como elemento del currículo se enmarca en el entorno social inmediato en el que se desenvuelve el alumno como sujeto de aprendizaje.

El entorno aporta el material cultural requerido para que el currículo dé respuesta a las necesidades, intereses y expectativas de la comunidad a la que pertenece el estudiante. Su importancia consiste en que el alumno logra alcanzar una adecuada comprensión de la propia cultura y una integración efectiva a su medio social.

En relación con lo anterior, Bolaños afirma:

“... no debe darse una relación unidireccional, en la cual la escuela recibe de la comunidad los aportes para enriquecer el currículo, sin aportar ella nada. Por el contrario, la escuela debe aprovechar su papel como institución social y cooperar en el fortalecimiento de los valores culturales del grupo. En este sentido, mediante el trabajo curricular y las acciones de proyección (campañas de divulgación cultural, reuniones, exposiciones, etc.) la escuela debe estimular a la comunidad para que revalore sus propias características, analice su realidad socio-cultural e inicie acciones para fortalecer y mejorar su propio entorno social" (1993, p. 42).

La interacción escuela-comunidad debe tener como consecuencia, la adquisición de aprendizajes significativos en el educando. En este sentido, la escuela unidocente debe garantizar la interrelación del contenido proveniente de la cultura sistematizada, con el contenido que subyace en el ambiente sociocultural. Desde una perspectiva ausbeliana, cuando un contenido sistematizado logra entroncarse con las experiencias previas del alumno, con los conceptos anteriormente adquiridos en su entomo, puede obtener un nuevo significado para la vida. Este principio está estrechamente relacionado con el aprendizaje combinatorio, según la teoría de aprendizaje significativo de David Ausbel.

La aportación de D. Ausbel se centra en la investigación en torno a cómo las personas reconstruyen continuamente su conocimiento, de que forma aprenden y 
de cuáles estrategias didácticas facilitan el proceso. En consecuencia, el aprendizaje significativo tiene lugar cuando las nuevas informaciones pueden relacionarse en forma no arbitraria con lo que la persona ya sabe.

Según Torres:

"En el momento en que aquello que se está aprendiendo puede ponerse en relación e integrarse con conocimientos que ya se poseen, es posible incorporarlo a las estructuras de conocimiento actuales. Por el contrario, cuando quien aprende se encuentra con contenidos que contempla sin apenas sentido, arbitrariamente conecta entre sí, y con verdaderas dificultades para relacionarlos con los contenidos de su actual estructura cognoscitiva, se produce un aprendizaje memorístico".

De esta manera, desde esta teoría de aprendizaje significativo, se requiere que el alumno posea con anterioridad algunas ideas inclusorias relacionadas con el contenido de las tareas escolares en que participa. Estas ideas son adquiridas en interacción con el medio y a partir de éstas adquiere significado el nuevo material. A este proceso se le llama "INCLUSIÓN", y en él se da la apropiación e inclusión de proposiciones potencialmente significativas en ideas más amplias y generales de la estructura cognoscitiva existente. Esto, a su vez. permite la organización jerárquica de la estructura cognoscitiva. Una vez que el alumno vivencia este proceso, puede ser capaz de comprender los contenidos a partir de una visión más holística e integradora.

Ausbel se refiere a este aspecto de la siguiente manera:

"La eficacia del aprendizaje inclusivo probablemente se pueda atribuir al hecho de que una vez que las ideas inclusivas se establecen adecuadamente en la estructura cognoscitiva... organizan nuevos hechos relacionados en torno a un tema común, con lo que se integran los elementos componentes del conocimiento nuevo tanto recíprocamente como con el conocimiento existente" (Ausbel, 1993 p. 62).

En este proceso de interrelación e integración cobra gran significación el principio teórico que Ausbel denomina "RECONCILIACIÓN INTEGRADORA". Para él, el acto de reconciliar ejerce una gran influencia en el aprendizaje, y en el contexto de su teoría este principio implica un nivel de profundidad mayor porque para su aplicación el alumno requiere de un mayor esfuerzo mental, al poner en ejercicio operaciones cognoscitivas que lo obligan a pensar.

En una interpretación de la teoría de Ausbel, Abarca (1993) se refiere a la reconciliación integradora en los siguientes términos: 
"Este principio incluye la capacidad para hacer referencia cruzada de ideas, reconciliar datos o hechos que aparentemente no están conectados, encontrar vínculos en toda la información disponible, explorar relaciones, hacer referencia a situaciones que se vieron tempranamente en el proceso del aprendizaje y que guardan relación con el concepto o con el conocimiento que se está aprendiendo" (Abarca, 1993, p. 89).

En el marco de este principio, se requiere que el estudiante sea capaz de captar la totalidad y no las partes de un curso de contenidos. Por ende, es necesario fortalecer una visión holística de pensamiento que facilite a y los alumnos, las alumnas y los docentes la interrelación de grandes cuerpos de conocimiento, para captar así su significado. En relación con este estilo de pensamiento, Abarca (1993) se refiere a él en el sentido de que los alumnos son capaces de aprender la totalidad para procesar y codificar contenidos, centrándose en aspectos fundamentales y no tomando tanto interés en los detalles.

La Política Educativa hacia el siglo XXI hace referencia explícita a la necesidad de orientar la educación hacia la multidisciplinariedad y la transdisciplinariedad, como respuesta a la preocupación ante el devenir histórico, pues las próximas generaciones enfrentarán un período de transición, caracterizado por nuevas preocupaciones en el campo del desarrollo tecnológico, el uso irracional de los recursos naturales, una visión deshumanizante y deshumanizada del desarrollo, el incremento del capital físico como fuente única de riqueza, la búsqueda de estrategias de desarrollo y competencia internacional, y la búsqueda de una concepción espiritual-humanista y humanizadora y la sostenibilidad de los recursos naturales, etc.

En consecuencia, la educación debe garantizar la formación de educandos con capacidad para resolver cualquier situación desde una perspectiva integral, con el fin de dar respuesta a estas apremiantes necesidades. En los considerandos de la Política Educativa se afirma:

"En resumen, el cambio de paradigma en la transición hacia el siglo XXI se caracteriza por una concepción de 'transdisciplinariedad', en donde las disciplinas de naturaleza científica tradicional y las ciencias sociales y humanas se interconectan y se afectan mutuamente. El dominio de las disciplinas es un paso necesario para manejar la transición hacia formas innovadoras de correlación e integración del conocimiento, hacia una ética del desarrollo y a un humanismo renovado que ubique al ser humano en el centro de las tareas del desarrollo y de la búsqueda del conocimiento" (Política Educativa hacia el siglo XXI 1994, p. 3). 
Esta concepción transdisciplinaria afecta necesariamente la práctica educativa. En este sentido, esta propuesta busca alcanzar los niveles de interdisciplinariedad y multidisciplinariedad. Una de las declaraciones de la Política Educativa hace hincapié en los efectos que tendría esta conceptualización en la práctica educativa:

"Los elementos que constituyen la PRÁCTICA EDUCA'TIVA: los planes y programas de estudio, los textos y otros recursos didácticos; los procesos de evaluación; la mediación del docente y la formación, capacitación y educación continua de los educadores. deben partir del Énfasis en las disciplinas; transcurrir hacia un enfoque multidisciplinario o correlacionada en donde cada disciplina contribuye a la construcción y reconstrucción del conocimiento sin perder la identidad propia para, finalmente, cuando sea apropiado, evolucionar hacia un enfoque transdisciplinario o de convergencia en donde cada disciplina Metodológicamente complemente e ilumina a otras" (Política Educativa 1994, p. 12).

En el intento de procurar reafirmar un estilo holístico de pensamiento en el alumno, sin que esto suponga un detrimento de las capacidades analíticas en el proceso de aprendizaje, resulta de primera importancia una readecuación curricular, a partir de los presupuestos teóricos que sustentan el CURRÍCULUM INTEGRADO.

Pocos alumnos son capaces de vislumbrar algo que facilite la unión o integración de los contenidos o el trabajo en general, de las diferentes asignaturas. La secuencia lógica con que se planifican los contenidos en los programas es difícilmente visible para el alumnado y en ocasiones para el mismo profesorado.

Según Torres (1995), el currículum puede organizarse, no sólo centrado en asignaturas, como ha sido tradicional. sino que puede planificarse alrededor de núcleos superadores de las disciplinas, centrados en temas, problemas, tópicos, instituciones, períodos históricos, espacios geográficos, colectivos humanos, ideas.

Esta idea de globalidad, de integración resulta necesaria porque en el mundo actual, pese a las grandes distancias geográficas que separan continentes y países, ya nada está aislado. Con los avances científico-tecnológicos nuestro planeta es un mundo global en el que todo se encuentra relacionado, tanto en el ámbito local, nacional e internacional. Un mundo caracterizado por la interdependencia financiera, política, cultural, ambiental y científica. Ninguno de estos aspectos podría ser comprendido satisfactoriamente al margen de los demás. 
Desde esta perspectiva, el currículum integrado se convierte asi en una alternativa capaz de correlacionar e integrar diversos contenidos a partir de grandes ejes conceptuales, facilitándose de esta manera la idea de globalidad.

Según Torres (1995),el carácter global de la percepción infantil de su entorno inmediato condiciona su desarrollo.

Él sostiene que los niños y las niñas no captan inicialmente las cosas por sus detalles y partes aisladas --que irian uniendo unas a otras hasta obtener una imagen de un objeto-, sino por el contrario, desde su globalidad. Pedagogos como Ovidio Decroly (1965) sostienen que los centros de interés son el resultado de esa percepción global de objetos, hechos y situaciones, que despiertan la curiosidad de niños y niñas.

Los centros de interés serían, por tanto, las ideas eje en torno a las que confluyen las necesidades fisiológicas, psicológicas y sociales del alumno.

Vygotski, en su teoría histórico-cultural, subraya el valor del aprendizaje, considerándolo como un proceso profundamente social, en el que se necesita adaptar las estrategias y contenidos de los proyectos curriculares al contexto histórico y cultural específico en el que vive el alumnado. Esta sería la forma en que se podrian llegar a utilizar los conceptos que posee y que son fruto de sus interacciones cotidianas en su medio social, con los nuevos conceptos que la escuela facilita. Es necesario, por tanto, crear situaciones de enseñanza y aprendizaje en las que se pueda dar la interacción de contenidos culturales previamente relacionados y los contenidos en el programa.

En la mayoría de los casos, las familias rurales no ofrecen la oportunidad de continuar en el sistema educativo con la educación secundaria y es por ello que ese tiempo debe ofrecer las mejores oportunidades a estos pobladores.

La interdisciplinariedad del conocimiento es una característica fundamental del presente siglo, es la reorganización del conocimiento. Torres (1995) afirma que este proceso de reorganización se fundamenta en tres grande dinámicas: una de ellas es la consecuencia lógica del trabajo científico e investigador que se desarrolla en especialidades concretas cayendo en la especialización, y generándose así determinadas parcelas temáticas.

Otra dinámica se relaciona con el campo de las disciplinas que comparten objetos de estudio, es decir, parcelas de un mismo tema, que genera así nuevos e interdisciplinarios ámbitos de conocimiento. Una tercera dinámica la constituye el surgimiento de equipos de investigación claramente interdisciplinarios, que han dado origen a centros, institutos y fundaciones que tienen como objetivo tratar de comprender y solucionar problemas de candente actualidad.

Esta tercera dinámica es la que mayor impacto presenta en la actualidad: 
"La complejidad del mundo y de la cultura actual obliga a desentrañar los problemas con múltiples lentes, tantas como áreas de conocimiento existen; de lo contrario, es fácil que los resultados se vean afectados por las deformaciones que impone la selectividad de las perspectivas de análisis a las que se recurre" (Torres 1995, p. 47).

Esta propuesta de integración curricular, entonces, parte del hecho de que la comprensión de cualquier suceso humano está siempre entrecruzándose por diversas dimensiones, es decir, es multicausal. Para Torres (1995), la cultura. mentalidad y expectativas de cualquier persona son fruto de una historia vivida en el seno de una o varias familias y es el resultado de su propia participación dentro de colectivos sociales; es imperativo tomar en cuenta informaciones relativas a todas esas dimensiones.

Diferentes disciplinas proporcionan esas informaciones. Se entiende por disciplina, una manera de organizar y delimitar un territorio de trabajo, de concentrar la investigación y las experiencias dentro de un determinado ángulo de visión. Desde esta concepción, cada disciplina nos of rece una imagen particular de la realidad, es decir, del objeto de estudio de su competencia. En consecuencia, una condición fundamental para que haya interdisciplinariedad es que existan disciplinas.

Por interdisciplinariedad se entiende el acto mediante el que se da la integración o función entre parcelas de disciplinas diferentes pero que comparten un mismo objeto de estudio (Torres, 1995).

Para efectos de la presente propuesta curricular, se toman en cuenta algunos de los criterios de reagrupación disciplinar, propuestas por Torres. Entre ellas están:

- Reagrupación de una o varias disciplinas de orientación teórica con una o varias de orientación práctica. Ejemplo: Eje Comunicación, ambiente y calidad de vida.

- Reagrupación de disciplinas que tengan homogeneidad marcada (puramente teórica o puramente práctica). Ejemplo: Ambiente y calidad de vida, con homogeneidad teórica.

Con base en el grado de integración de las diversas áreas o asignaturas, para esta propuesta se ha optado por retomar la jerarquización de niveles de colaboración e integración disciplinar, propuestos por Jean Piaget. Estos son: 
1. Multidisciplinariedad: es un nivel inferior de integración. Ocurre cuando, para solucionar un problema se busca información y ayuda en disciplinas, sin que dicha interacción contribuya a modificarlos o enriquecerlos.

2. Interdisciplinarias: este nivel de asociación se da entre disciplinas, cuando la cooperación entre algunas de ellas lleva a interacciones reales, es decir. hay una verdadera reciprocidad en los intercambios y por consiguiente. enriquecimientos mutuos (Piaget, 1979, pp. 166-71).

Según Torres (1995), para Jean Piaget, la finalidad de la investigación interdisciplinaria consiste en procurar una recomposición o reorganización de los ámbitos del saber.

En la dinámica de esta reorganización y en interacción constante entre el alumno y el objeto de estudio, la finalidad inmediata se orienta a que estudiante y docente visualicen en cada eje los contenidos como un medio que les permita trascender la rigidez conceptual, para llegar a procesos educativos que enfaticen tanto lo cognitivo como lo socioafectivo y lo psicomotor. De esto se desprende que el papel del docente adquiere una significación particular y decisiva en la formación del alumno, porque le corresponde orientar el desarrollo del contenido en una dimensión de integralidad, trascendiendo lo memoristico y arbitrario para fortalecer una mediación docente en la que se rescate lo actitudinal y valorativo, en procura de una mejor calidad de vida.

El educador le corresponde planificar las expectativas de aprendizaje de manera que tengan coherencia y unidad, es decir, que se visualice adecuadamente una secuencia de aprendizaje en cada una de las diferentes áreas y ejes temáticos. El educado, entonce, deberá seleccionar aquellos objetivos de las diferentes áreas, que tengan correlación entre sí, y a partir de ahí, seleccionar las diversas actividades que completen una secuencia según sea el área de estudio. En el desarrollo de actividades deberá tener cuidado de incorporar aquellos elementos de la comunidad que puedan tener relación con el tema en estudio. En este sentido, tratará continuamente de integrar la comunidad al currículo escolar y la escuela a la comunidad, proyectándola en aquellos aspectos y acciones determinantes del desarrollo comunal.

Al alumno le corresponde convertirse en el protagonista, porque debe actuar en una dinámica de constante interrelación con su medio sociocultural. Esta interrelación debe permitirle apropiarse, tanto de los contenidos de la cultura sistematizada, como de los provenientes de su entorno inmediato. Le corresponde, entonces, captar e interiorizar la correlación que irá encontrando en las diferentes asignaturas y aprenderlas de manera significativa. 
Al alumno le corresponde convertirse en el protagonista, porque debe actuar en una dinámica de constante interrelación con su medio sociocultural. Esta interrelación debe permitirle apropiarse, tanto de los contenidos de la cultura sistematizada, como de los provenientes de su entorno inmediato.
Debe tener capacidad para compartir y negociar significados en el trabajo cooperativo, tener iniciativa, responsabilidad individual, participar con entusiasmo, ser creativo, respetuoso de la diversidad cultural, social y étnica, pero sobre todo, cultivar el bien común.

Una tarea importante de los alumnos es realizar procesos intelectuales que les permita aprender a aprender, a pensar y a com-

prender; que les permita desarrollar su pensamiento e integrarse satisfactoriamente en la dinámica social de la comunidad.

Y, por último, es preciso mencionar que los niños y las niñas deben mantener relaciones de apoyo mutuo entre sí. Para este efecto, aquellos y aquellas que manifiestan mayor habilidad, interés o capacidad en alguno de los ejes integradores, ya sea en el área intelectual, socioafectiva o psicomotora. podrán convertirse en tutores para sus compañeros y compañeras de su nivel y de otros niveles inferiores.

En relación con el contenido, es necesario aclarar que éste no pierde su importancia en el marco de la presente propuesta. Lo que sí es necesario recalcar es el hecho de la nueva connotación con que debe mirársele, pues deja de ser un fín en sí mismo, como tradicionalmente sucede, para convertirse en un medio de realización integral del educando.

El tratamiento que se le da a los contenidos parte de una posición en la que se desarrolla un tema, pero inmediatamente se establecen relaciones y referencias concretas con la vida misma del educando, con la realidad o con un problema o situación determinado que puede derivarse de su entorno inmediato o del entorno comunal.

En relación con la evaluación, interesa evaluar formativamente a lo largo del proceso de aprendizaje, con el fin de determinar si existe comprensión y apropiación del conocimiento por parte del alumno y la alumna.

Para obtener esta valoración pueden plantearse preguntas, para que los alumnos y las alumnas expliquen, relacionen o reconstruyan conceptos determinados.

Debe evaluarse la forma en que ellos y ellas realizan el abordaje de nuevos contenidos provenientes del entorno comunal, asimismo, la forma en que se enfrentan a un problema o tarea específica. 
Partiendo del marco de referencia vigente se retoma también la evaluación diagnóstica y sumativa. Para esta última se propone una evaluación por ejes integradores, donde se tome en cuenta las inteligencias múltiples que expone Haward Gardner, quien las relaciona con la capacidad de resolver problemas y hacer productos en un contexto rico de ambientación naturalista. Entre más contextos se incluyan para valorar en cada eje temático, más se estará ayudando a las y los estudiantes a descubrir su talento en un área específica. Si bien el alumno y la alumna pueden mostrar competencia en más de un talento, siempre habrá alguno en que se destaquen más. Para esta valoración se propone el uso del portafolio.

En general, la presente propuesta pretende impulsar en las escuelas unidocentes, una educación interdisciplinaria porque es un medio adecuado para enfrentar satisfactoriamente la problemática que generan los ágiles cambios de la sociedad actual. En este aspecto, Torres expresa:

\begin{abstract}
"Alumnos y alumnas con una educación más interdisciplinaria están más capacitados para enfrentarse a problemas que trascienden los limites de una disciplina concreta y para detectar, analizar y solucionar problemas nuevos con los que nunca antes se han visto. La motivación para el aprendizaje es muy grande ya que cualquier situación o problema que preocupe o interese a los estudiantes puede convertirse en objeto de estudio" (1995, p. 75).
\end{abstract}

Se hace urgente un cambio cualitativo en la escuela, el cual permita la formación de una docente y de una alumna, con una nueva mentalidad y una actitud frente a la vida. Desde esta óptica, el aula se convierte en la microcultura que genera ese cambio, porque en ella debe reproducirse el ambiente de la comunidad.

Las experiencias que se viven en familia y en comunidad deben ser analizadas, mediante procedimientos que favorezcan actitudes de respeto, tolerancia, diálogo y solidaridad. En el aula deben propiciarse el desarrollo del espíritu creativo y la actitud crítica frente a la realidad. Deben verse y analizarse lo cotidiano en interacción con el contenido de materia.

La escuela, en asociación con otras entidades locales, puede propiciar:

- El aprovechamiento de los recursos de la comunidad.

- La identificación de cada docente con la problemática de la escuela y la comunidad.

- La integración y participación de los miembros de la comunidad en la discusión de problemas.

- La inserción de la escuela en su realidad social.

- El apoyo a las entidades locales. 
En este sentido, puede dilucidarse que los procesos educativos, además de favorecer el desarrollo personal en lo biológico, lo afectivo y lo psicomotor, también deben establecer compromisos con el desarrollo social y comunitario.

En términos generales, la opción de esta propuesta gira en torno a la multidisciplinariedad y la interdisciplinariedad, además del desarrollo integral del niño y la niña, y a rescatar e impulsar la misión de que la escuela es la institución más cercana al corazón de la familia y de la comunidad.

Un proyecto educativo fundamentado en la integración del currículum y adscrito a una epistemología constructiva debe, en materia evaluativa, responder a un paradigma alternativo como el naturalista como medio propicio para valorar en toda su magnitud, los eventos de construcción y de reconstrucción del conocimiento tanto por parte del mediador como de los mismos educandos.

Una praxis, como la educativa, inmersa en el ámbito de las Ciencias Sociales donde existen tantas realidades como grupos humanos, por ser éstas construcciones mentales, debe ejecutarse y valorarse partiendo del cambio y lo diverso en vez de lo estático y lo uniforme.

Una evaluación para que pueda responder y atender las particularidades cognoscitivas, afectivas y motrices tan disímiles de un espacio áulico a otro, debe caracterizarse por ser, entre otros aspectos, flexible, dialógica y también una situación de aprendizaje a la vez.

Asumirla como flexible permitirá atender las diferencias innegables que hay entre los educandos. Cada uno podrá progresar a su ritmo y se acreditará su nivel de aprovechamiento con respecto a éste y de acuerdo con sus limitaciones o potenciales.

Darle una dimensión dialógica garantiza que, simultáneo a los eventos de construcción y de reconstrucción del conocimiento, haya un permanente intercambio de opiniones de los sujetos directamente involucrados en el proceso que fortalecería y realimentaría al mismo.

Como espacio de aprendizaje, la evaluación así asumida propiciaría la metacognición de los involucrados y las involucradas al poder éstos y éstas exteriorizar ¿cómo han llegado al conocimiento?, ¿qué dificultades encontraron?, ¿cómo las superaron?, ¿por qué les resulta significativo o no? $\mathrm{Y}$ ¿qué aplicabilidad le ven para su quehacer cotidiano o para su futuro?

Para asumir la evaluación desde esta perspectiva debe recurrirse a una técnica no tradicional de obtener información. Entre las posibles, el Portafolio de evaluación dinámica es la más apropiada, por ser este un espacio donde los educandos exhiben descubrimientos, progresos, esfuerzos, mientras externan sus sentimientos y vivencias sobre los mismos. 
Al permitir la evaluación por medio del portafolio, que los educandos den cuenta de sus estrategias y procesos que determinaron sus éxitos y fracasos, les permite a los mediadores explotar más la función formativa de la evaluación para replantear y realimentar las situaciones de aprendizaje que les proporciona a los educandos.

El portafolio, como han mencionado Garcia, J. y Jiménez, K. “...permite a los participantes tener conciencia de sus propios procesos cognitivos, de aprendizajes, de significación de las experiencias vividas y de las reflexiones surgidas."

Sobre esta técnica el Departamento de Evaluación del MEP, en el documento Orientaciones teórico-prácticas para mejorar el proceso de evaluación de los aprendizajes, manifiesta: "el portafolio reúne evidencias de parte de los estudiantes para demostrarse y demostrar que han aprendido y cómo lo han logrado" (s. f.).

Aparte de ser una técnica confiable, brinda otras posibilidades para conocer aspectos afectivos, asi como el tipo de aprovechamiento que tienen las y los estudiantes sobre el proceso mismo de la mediación pedagógica.

Es una técnica, de múltiples posibilidades que como han subrayado García, J. y Jiménez, K. se caracteriza por ser:

“...un recurso flexible que permite documentar experiencias personales y grupales, además propicia la sistematización de las experiencias desde una perspectiva dinámica, concreta y que responde a la realidad del sujeto. En este sentido se puede afirmar que el portafolio incorpora diversidad de aspectos significativos de la cotidianidad de los participantes".

Para la evaluación concreta, en este Proyecto, se recurrirá a las tres funciones de la evaluación que puntualiza el Marco de referencia y directrices técnicas para la evaluación en el sistema de educación formal: la diagnóstica, la formativa y la sumativa.

La formativa será la que más se explote por la particularidad misma de la técnica del portafolio que requiere realimentación permanente. El portafolio será la estrategia evaluativa predominante, tendrá por ello un peso porcentual de un 75\%. Entre sus principales criterios de evaluación contará con los siguientes: progreso del y la estudiante, aprobación de conocimientos, capacidad de síntesis, creatividad, integración de conocimientos y aquellos que negocie cada docente con sus alumnas y alumnos.

El $25 \%$ de la evaluación se obtendrá de la función sumativa, una prueba comprensiva al finalizar cada periodo. El propósito de esta prueba es para que las y los estudiantes, a la hora de incursionar el tercer ciclo o en la eventualidad 
de que se trasladen a otros centros educativos, no se extrañen ante una evaluación por medio de pruebas.

\section{Objetivos}

Sus objetivos se pueden resumir como la contribución al mejoramiento de la calidad de la educación en las escuelas unidocentes para que las alumnas y los alumnos construyan aprendizajes significativos y permanentes en interacción con el medio potenciando su desarrollo.

\section{La experiencia}

Se ejecutó durante cuatro años. Los dos primeros años en catorce escuelas del circuito 04 de la Dirección Regional de Guápiles y en el tercero y cuarto se amplió a veinticinco escuelas incluyendo dos de la Dirección Regional de Limón.

A solicitud de la Dirección Regional de Pérez Zeledón se desarrolló la experiencia con dieciséis escuelas durante el año 1999.

\section{Estrategia metodológica}

Para la elaboración de la propuesta de contextualización curricular se hizo un análisis de los contenidos de las asignaturas que se estudian en $\mathrm{I}^{\circ}$ y $11^{\circ}$ ciclos, para reorganizarlos en un mesoplaneamiento: que es un nuevo documento que reorganiza los contenidos de los programas de estudio alrededor de ejes integradores, que responden a la pregunta:

¿Para qué sirve este contenido en esté contexto?

(comentarios Académicos en Desarrollo Regional y Rural. N³, agosto. 1997).

Estos ejes integradores que organizan la relación entre el currículo sistematizado y el cotidiano, para orientar la forma de planear la mediación pedagógica del aprendizaje y la enseñanza son:

1. Comunicación: incluye los contenidos del programa de español; van dirigidos hacia el logro de una persona capaz de comunicarse con los demás. Es necesario que maestros, estudiantes, padres y madres, valoren la comunicación como una herramienta importantísima para resolver los problemas de la familia 
y la comunidad. Se hace énfasis en la expresión oral, la escucha, la lectura y la escritura como base para la adquisición de los conocimientos de todas las áreas del saber, así como la práctica de actividades recreativas y lúdicas del lenguaje.

2. Ambiente y calidad de vida: incluye los contenidos del programa de estudios sociales y parte de los contenidos de los programas de ciencias y agricultura. En este eje es fundamental conceptualizar la escuela insertada en la comunidad y cada niña y niño proyectando en ella su realidad familiar, con base en la cual hay que ejemplificar los contenidos sistematizados, para darle significado al aprendizaje. Aquí se problematiza el currículo haciendo lectura de la realidad en que se encuentra la escuela, propiciando el reconocimiento de los problemas, crcando soluciones y que la superación está ligada a una mejor calidad de vida; para entender que todos somos copartícipes del desarrollo de nuestra comunidad, del distrito, del cantón, del país y, por consiguiente, del mundo.

3. Pensamiento lógico matemático: incluye los contenidos del programa de matemática. Es uno de los retos más importantes de la propucsta, se busca que esta asignatura se convierta en una herramienta que puede ser utilizada para una mejor comprensión de los otros ejes, relacionando cada contenido con el contexto que rodea al estudiante; particndo de lo concreto para construir las estructuras mentales que permitan la abstracción de otros conceptos que desarrollan la capacidad de razonamiento para la vida. Al respecto afirma De Zubería.

“...el desarrollo del pensamiento y la capacidad intelectual dependen única y exclusivamente de la calidad y cantidad de los conceptos y de las operaciones intelectuales que domina y manipula un ser humano ... ejercitar al máximo sus Operaciones Intelectuales producirá individuos muy dotados intelectualmente: atletas de la inteligencia".

4. Pensamiento científico: incluye parte de los contenidos del programa de ciencias y agricultura. Tiene como reto inculcar en las y los estudiantes el principio de que la tecnología debe ser compartida por todos los países y debe ser utilizada solamente en beneficio de la humanidad. El enfoque de la ciencia debe enriquecer el pensamiento y cultivar en él las habilidades y actitudes que le permitan descubrir, apreciar y usar los conocimientos científicos, por lo que los procesos de observación, medición, clasificación, experimentación deben orientar la práctica cotidiana de esta propuesta.

Para operativizar la propuesta fue necesario variar el desarrollo tradicional de las clases; por lo que se propuso una mediación pedagógica que facilite los procesos de aprendizaje con temas relacionados para los diferentes niveles, de acuerdo con la secuencia que aparece en los programas de estudio, sólo que 
aumentando el grado de profundidad en cada nivel; el desarrollo de las actitudes y habilidades expresadas en los ejes integradores; la práctica de valorcs en la convivencia cotidiana y la integración de la comunidad a la cscuela. Esta mediación pedagógica se ejecuta en cuatro momentos:

- Actividades iniciales: este primer período se trabaja con todos y todas las estudiantes de los diferentes niveles en actividades relacionadas con la música, la educación física, el arte, la autoestima, los valorcs o cualquier otro aspecto que fomente en las alumnas y los alumnos el crecimiento personal y espiritual, la reflexión, el desarrollo integral y la creatividad. Además se organiza el quehacer educativo del día.

- Trabajo grupal por nivcles: este scgundo período desarrolla trabajo grupal por niveles en cl estudio de los ejes ambiente, calidad de vida y comunicación.

- Trabajo grupal por niveles: en cste tercer período se desarrolla un trabajo grupal por niveles en el estudio de los ejes pensamiento logico-matemático y pensamiento científico.

- Reconstrucción de experiencias: en este cuarto período de nuevo se encuentran las y los estudiantes de los seis niveles para exponer ante sus compañeras y compañeros los aprendizajes obtenidos. En cste período se pueden realizar plenarias, exposiciones, dramatizacioncs, murales, dibujos, trabajos creativos utilizando la mayor variedad de técnicas que pernitan dinamizar al máximo el aprendizaje. También en forma individual se sistematizan los aprendizajes en un cuaderno de experiencias, que fomenta la expresión escrita alrededor de las percepciones y los sentires particulares, para compartirlos a través de la lectura y con ello la utilización de la escritura con significado.

Otro de los aspectos que requieren cambios acordes con la propuesta es la evaluación, pues debe ser más cualitativa y promovedora de sentimientos de éxito, motivación, metacognición. Por ello la función diagnóstica, formativa y sumativa está presente cn forma constante.

Propiciar el encuentro entre adultos de la comunidad y la población escolar fue un reto que tomo forma en una actividad complementaria que se nombro como Talleres de oficios. Este espacio se construye con la participación de los adultos y adultas de la comunidad en tomo a oficios de la cultura rural que han posibilitado el desarrollo de soluciones a las diferentes necesidades de las familias.

Es una forma de integrar al currículum escolar, las raíces y los valores que han creado arraigo e identidad de una forma de vida con sus propias características y que son parte del origen del país y del ser costarricense. Un taller de oficio, 
muy importante, es el cultivo de la tierra a través de la huerta escolar e integrando alrededor del proceso la formación nutricional en los comedores escolares.

La última actividad que se propuso para fortalecer la experiencia de cada escuela y comunidad fue el Festival de Escuelas, donde se busca la proyección de las diferentes experiencias y la consolidación de las habilidades de todas y todos los y las participantes, en escenarios mucho más amplios, que lo que ofrece el espacio cotidiano de la escuela, por lo que la persona adquiere mayor confianza y seguridad para desempeñarse en cualquier lugar que la vida le ofrezca.

\section{RELACIÓN DE LA EDUCACIÓN FORMAL CON EL DESARROLLO RURAL}

Si las instituciones educativas ofrecen experiencias de aprendizaje que fortalezcan la autoestima, la identidad, la comprensión crítica de la realidad y el desarrollo de propuestas que la modifiquen, se estará gestando personas más seguras, con arraigo, que sentirán orgullo y satisfacción de pertenecer a un país cuya riqueza primordial es la ruralidad desde la historia y en la actualidad.

La educación básica así, es una inversión a largo plazo que requiere visión y compromiso desde todos los aspectos: políticos, económicos, sociales.

Así la escuela se convierte en el espacio donde predomina la práctica del trabajo cooperativo, la capacidad de diálogo, el respeto por las ideas propias y ajenas, aprendiendo a tomar decisiones individuales y colectivas y a organizarse en lo cotidiano para resolver tareas específicas, que desarrollen el carácter y la voluntad, unido al arraigo con autoestima, de los (as) futuros (as) adultos (as) que liderarán el desarrollo sostenible de sus pueblos, modificando las múltiples realidades con soluciones que emerjan desde lo interno de las diferentes ruralidades que tiene Costa Rica.

Contextualizar el currículo no implica descartar algún contenido de los macroplaneamientos nacionales, al contrario es darle significado integrando el entorno, sus riquezas y dificultades al aprendizaje sistematizado que ofrece el sistema educativo nacional.

\section{Referencias}

Aguilar, M. E. y Monge, M. E. (1994). Hacia una pedagogía rural María Ester Aguilar y Marta Eugenia Monge, Heredia: ANDE.

Borsotti, C. (1984) Sociedad rural, educación y escuela en América Latina. Buenos Aires: Kapeluz. 
Camp, R. (1991) Portfolio reflection in middle and secondary school classrooms. The place of portfolio in our changing views of writing assessment. Assessment in the context of schools and shool change. N. Jersey: Educational Testing Scrvice. S.I: Inédito.

Colombia, Ministerio de Educación Nacional. (1991) Escuela de mostrativas y microcentros rurales. Bogotá: Ediciones Programas Escuela Nueva.

Costa Rica, Ministerio de Educación Pública. Depto. de Evaluación Educativa: Orientaciones teórico-prácticas para mejorar el proceso de evaluación de los aprendizajes. San José, C.R.: El Ministerio s.f.

Costa Rica, Ministerio de Educación Pública Español: I ciclo (1995).San José, C. R.: El Ministerio.

Costa Rica, Ministerio de Educación Pública Español: /l ciclo (1995). San José, C. R.: El Ministerio.

DeZubería, J. y González, M. A. (1995) Estrategias metodológicas y criterios de evaluación. Bogotá: Fundación Alberto Merani.

DeZubería. M. (1995). Formación de valores y actitudes: un reto a las escuelas de futuro. Bogotá: Fundación Alberto Merani.

DeZubería, M. (1994). Los modelos pedagógicos. Bogotá: Fundación Alberto Merani.

DeZubería, M. (1994) Pensamiento y aprendizaje: los instrumentos del conocimiento. Bogotá: Fundación Alberto Merani.

DeZubería, M. y DeZubería Rojo, A. (1995). Operaciones intelectuales y creatividad. Bogotá: Fundación Alberto Merani, 1995.

García, J. y Jiménez K. Portafolio de evaluación. S.I., s.n., s.f.

Kaplún, M. (1984). El comunicador popular. Caracas, V.E.: CIESPAL.

Molina Bogantes, Z. (1995). La contextualización del curriculo: una estrategia para alcanzar la calidad y la equidad en la educación. San José, C.R.: Ministerio de Educación Pública. 
Palmer Wolf, D. (1989). Portafolio assessment: sampling student work. Educational Leardership. 46 (7), april, 35-39. S.I., s.n.

Programa UNIR (1997). Comentarios Académicos en Desarrollo Regional y Rural. $\mathrm{N}^{\circ} 3$, agosto, UNA. EUNA.

Universidad Nacional (1991). Centro de Investigacion y Docencia en Educación. División de Educación Rural: Plan de desarrollo académico 92-97. Heredia, C.R.: DER.

Vega Posada, R. (1998). Conferencia electrónica (POBREZA, COMUNIDADES CAMPESINAS Y DESARROLLO RURAL "GOBIERNOS LOCALES Y DESARROLLO RURAL EN LOS ANDES: CASOS Y EXPERIENCIAS", PUNO. 